\title{
3. UNDERWAY GEOPHYSICS, GLOMAR CHALLENGER, LEG 78B 1
}

\author{
Matthew H. Salisbury, Deep Sea Drilling Project, Scripps Institution of Oceanography \\ Roy D. Hyndman, Pacific Geoscience Centre, Department of Energy, Mines and Resources, Sidney, B.C. \\ and \\ Barbara Long, Deep Sea Drilling Project, Scripps Institution of Oceanography²
}

While steaming from San Juan, Puerto Rico to Site 395, and from Site 395 to Las Palmas in the Canary Islands on Leg 78B, the Glomar Challenger towed a Geometrics V4970 proton-precession magnetometer and two Bolt airguns with 40- and 120-cubic-inch capacities. In addition, the bathymetry was monitored continuously with a $12.5-\mathrm{kHz}$ pinger. The ship's position was determined through a combination of dead reckoning and satellite navigation; the satellite fixes were obtained with a Magnavox MX702 satellite navigation system.

The ship's track listed in Table 1 and shown in Figure 1 is accurate to within one nautical mile (Talwani et al., 1966), and lies within a few tens of kilometers of the paths taken on Legs 45 and 46 (Rabinowitz et al., 1979). Within a few kilometers of the site, checks conducted during re-entry demonstrated that the navigational accuracy was improved to $\pm 100 \mathrm{~m}$ by paying increased attention to basement topography while approaching the site. The total-field magnetic anomaly shown in Figure 1

\footnotetext{
${ }^{1}$ Hyndman, R. D., Salisbury, M. H., et al., Init. Repts. DSDP, 78B: Washington (U.S. Govt. Printing Office).

2 Addresses: (Salisbury and Long) Deep Sea Drilling Project, Scripps Institution of Oceanography, La Jolla, CA 92093; (Hyndman) Pacific Geoscience Centre, Dept. of Energy, Mines and Resources, Sidney, B.C., Canada.
}

is plotted normal to the ship's track, with the track representing a magnetic anomaly of zero with respect to the 1980 International Geomagnetic Reference Field. A detailed plot of bathymetry and magnetic anomaly strength is plotted as a function of distance along the ship's track in Figure 2. Dates and times are also shown in Figure 2 for comparison with Figure 1. The airgun records and the individual readings of bathymetry and magnetic field strength on which these figures are based are not shown here, but may be obtained upon request from the National Geologic Data Center, Boulder, Colorado.

\section{ACKNOWLEDGMENTS}

We wish to express our appreciation to Gus Gustafson, the laboratory officer on the Glomar Challenger, and to the seagoing technical staff on Leg 78B, for their assistance in obtaining the underway data. We also thank Stuart Smith of the Underway Geophysical Data Center at Scripps Institution of Oceanography for data processing.

\section{REFERENCES}

Rabinowitz, P. D., Heirtzler, J. R., Aikens, T. D., and Purdy, G. M., 1979. Underway geophysical measurements: Glomar Challenger Legs 45 and 46. In Melson, W. G., Rabinowitz, P. D., et al., Init. Repts. DSDP, 45: Washington (U.S. Govt. Printing Office), 55-118.

Talwani, M., Dorman, L., Worzel, J. L., and Bryan, G. M., 1966. Navigation at sea by satellite. J. Geophys. Res., 71:5891-5902. 


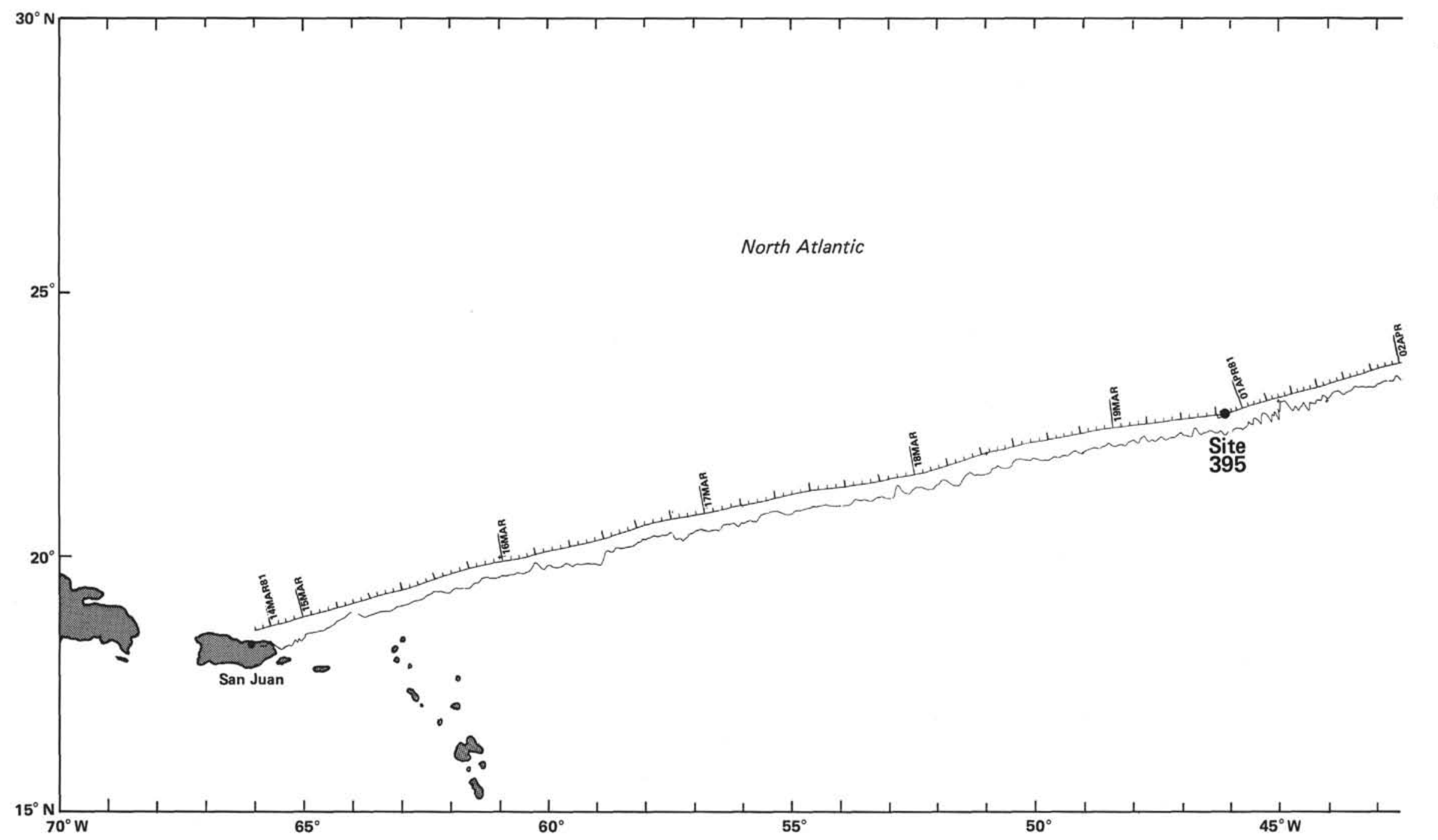




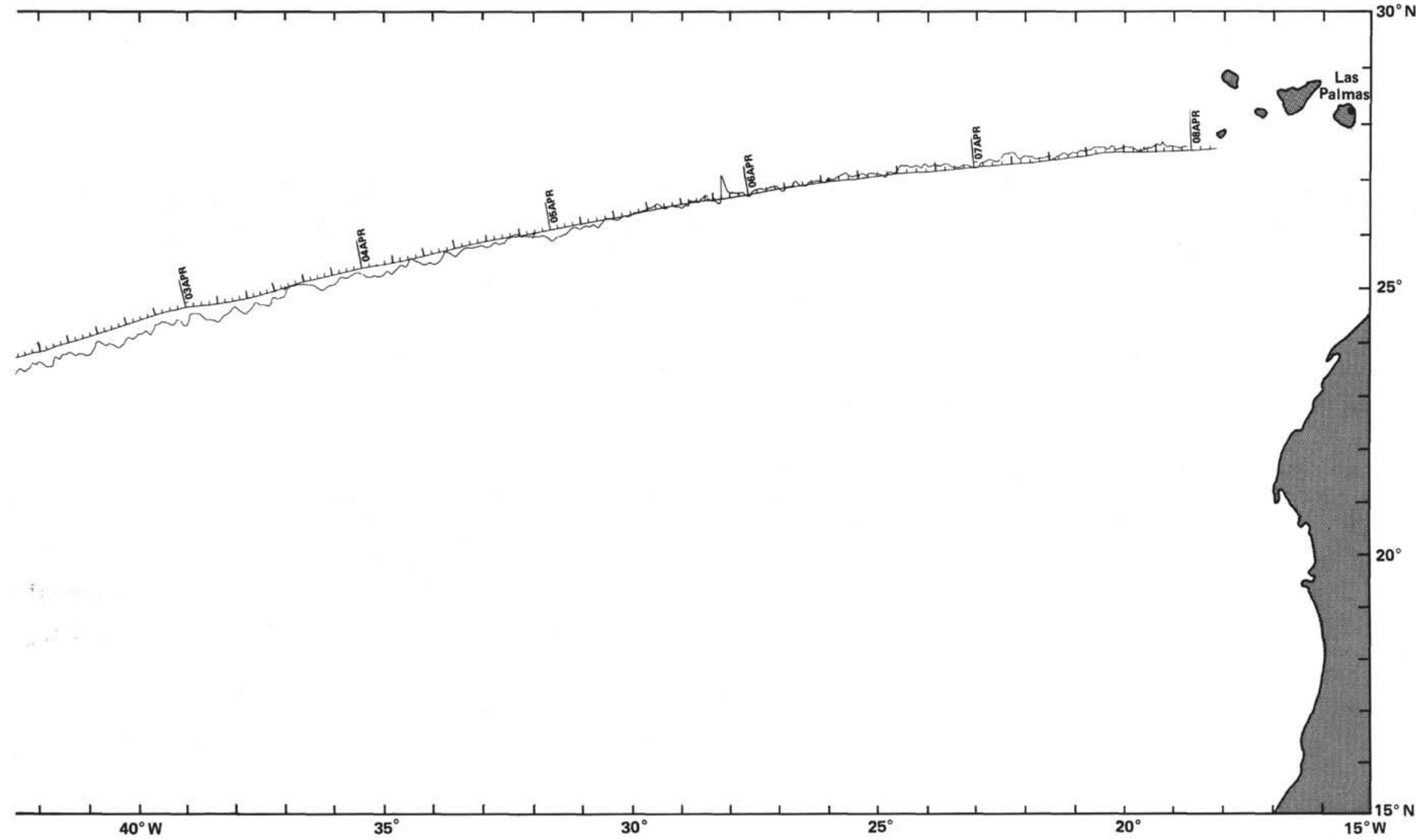

Figure 1. Glomar Challenger's track on Leg 78B. Also shown is the total-field magnetic anomaly as a function of position along the track (track $=$ zero anomaly). Ticks along track represent hour marks. 
Table 1. Leg 78B navigation data.

\begin{tabular}{|c|c|c|c|c|c|}
\hline Date & Month ${ }^{\mathrm{a}}$ & $\begin{array}{l}\text { Time } \\
\text { (GMT) }\end{array}$ & Latitude & Longitude & $\begin{array}{l}\text { Distance } \\
\text { (n. mi.) }\end{array}$ \\
\hline 14 & 3 & 1746 & $18^{\circ} 33.7^{\prime} \mathrm{N}$ & $66^{\circ} 1.3^{\prime} \mathrm{W}$ & 0.0 \\
\hline 14 & 3 & 1748 & $18^{\circ} 33.8^{\prime} \mathrm{N}$ & $66^{\circ} 1.1^{\prime} \mathrm{W}$ & 0.2 \\
\hline 14 & 3 & 1800 & $18^{\circ} 34.3^{\prime} \mathrm{N}$ & $65^{\circ} 59.3^{\prime} \mathrm{W}$ & 2.0 \\
\hline 14 & 3 & 2100 & $18^{\circ} 42.3^{\prime} \mathrm{N}$ & $65^{\circ} 31.0^{\prime} \mathrm{W}$ & 30.0 \\
\hline 14 & 3 & 2115 & $18^{\circ} 42.4^{\prime} \mathrm{N}$ & $65^{\circ} 28.6^{\prime} \mathrm{W}$ & 32.3 \\
\hline 14 & 3 & 2202 & $18^{\circ} 44.5^{\prime} \mathrm{N}$ & $65^{\circ} 21.2^{\prime} \mathrm{W}$ & 39.6 \\
\hline 14 & 3 & 2348 & $18^{\circ} 49.8^{\prime} \mathrm{N}$ & $65^{\circ} 3.0^{\prime} \mathrm{W}$ & 57.6 \\
\hline 15 & 3 & 000 & $18^{\circ} 50.3^{\prime} \mathrm{N}$ & $65^{\circ} 0.9^{\prime} \mathrm{W}$ & 59.7 \\
\hline 15 & 3 & 434 & $19^{\circ} 2.8^{\prime} \mathrm{N}$ & $64^{\circ} 12.5^{\prime} \mathrm{W}$ & 107.1 \\
\hline 15 & 3 & 500 & $19^{\circ} 4.0^{\prime} \mathrm{N}$ & $64^{\circ} 8.3^{\prime} \mathrm{W}$ & 111.2 \\
\hline 15 & 3 & 622 & $19^{\circ} 7.8^{\prime} \mathrm{N}$ & $63^{\circ} 55.1^{\prime} \mathrm{W}$ & 124.3 \\
\hline 15 & 3 & 950 & $19^{\circ} 17.5^{\prime} \mathrm{N}$ & $63^{\circ} 20.3^{\prime} \mathrm{W}$ & 158.6 \\
\hline 15 & 3 & 1136 & $19^{\circ} 21.3^{\prime} \mathrm{N}$ & $63^{\circ} 3.2^{\prime} \mathrm{W}$ & 175.1 \\
\hline 15 & 3 & 1157 & $19^{\circ} 22.1^{\prime} \mathrm{N}$ & $62^{\circ} 59.6^{\prime} \mathrm{W}$ & 178.6 \\
\hline 15 & 3 & 1336 & $19^{\circ} 26.5^{\prime} \mathrm{N}$ & $62^{\circ} 42.9^{\prime} \mathrm{W}$ & 195.0 \\
\hline 15 & 3 & 1354 & $19^{\circ} 27.5^{\prime} \mathrm{N}$ & $62^{\circ} 39.8^{\prime} \mathrm{W}$ & 198.1 \\
\hline 15 & 3 & 1450 & $19^{\circ} 31.0^{\prime} \mathrm{N}$ & $62^{\circ} 30.2^{\prime} \mathrm{W}$ & 207.8 \\
\hline 15 & 3 & 1522 & $19^{\circ} 32.8^{\prime} \mathrm{N}$ & $62^{\circ} 25.0^{\prime} \mathrm{W}$ & 213.0 \\
\hline 15 & 3 & 1541 & $19^{\circ} 33.9^{\prime} \mathrm{N}$ & $62^{\circ} 21.7^{\prime} \mathrm{W}$ & 216.3 \\
\hline 15 & 3 & 1556 & $19^{\circ} 34.7^{\prime} \mathrm{N}$ & $62^{\circ} 19.1^{\prime} w$ & 218.9 \\
\hline 15 & 3 & 1615 & $19^{\circ} 35.8^{\prime} \mathrm{N}$ & $62^{\circ} 15.9^{\prime} \mathrm{W}$ & 222.0 \\
\hline 15 & 3 & 1638 & $19^{\circ} 37.2^{\prime} \mathrm{N}$ & $62^{\circ} 12.0^{\prime} \mathrm{W}$ & 226.0 \\
\hline 15 & 3 & 1715 & $19^{\circ} 39.2^{\prime} \mathrm{N}$ & $62^{\circ} 5.7^{\prime} \mathrm{W}$ & 232.3 \\
\hline 15 & 3 & 1742 & $19^{\circ} 40.3^{\prime} \mathrm{N}$ & $62^{\circ} 1.0^{\prime} \mathrm{W}$ & 236.8 \\
\hline 15 & 3 & 2008 & $19^{\circ} 47.3^{\prime} \mathrm{N}$ & $61^{\circ} 36.3^{\prime} \mathrm{W}$ & 261.1 \\
\hline 15 & 3 & 2154 & $19^{\circ} 51.5^{\prime} \mathrm{N}$ & $61^{\circ} 18.0^{\prime} \mathrm{W}$ & 278.8 \\
\hline 15 & 3 & 2154 & $19^{\circ} 51.5^{\prime} \mathrm{N}$ & $61^{\circ} 18.0^{\prime} \mathrm{W}$ & 278.8 \\
\hline 16 & 3 & 000 & $19^{\circ} 55.1^{\prime} \mathrm{N}$ & $60^{\circ} 55.9^{\prime} \mathrm{W}$ & 300.0 \\
\hline 16 & 3 & 116 & $19^{\circ} 57.3^{\prime} \mathrm{N}$ & $60^{\circ} 42.5^{\prime} \mathrm{W}$ & 312.7 \\
\hline 16 & 3 & 148 & $19^{\circ} 58.1^{\prime} \mathrm{N}$ & $60^{\circ} 37.2^{\prime} \mathrm{W}$ & 317.8 \\
\hline 16 & 3 & 238 & $19^{\circ} 59.9^{\prime} \mathrm{N}$ & $60^{\circ} 29.0^{\prime} \mathrm{W}$ & 325.7 \\
\hline 16 & 3 & 300 & $20^{\circ} 0.7^{\prime} \mathrm{N}$ & $60^{\circ} 25.1^{\prime} \mathrm{W}$ & 329.4 \\
\hline 16 & 3 & 344 & $20^{\circ} 2.6^{\prime} \mathrm{N}$ & $60^{\circ} 17.9^{\prime} \mathrm{W}$ & 336.4 \\
\hline 16 & 3 & 428 & $20^{\circ} 4.4^{\prime} \mathrm{N}$ & $60^{\circ} 10.2^{\prime} \mathrm{W}$ & 343.9 \\
\hline 16 & 3 & 530 & $20^{\circ} 7.0^{\prime} \mathrm{N}$ & $59^{\circ} 59.4^{\prime} \mathrm{W}$ & 354.4 \\
\hline 16 & 3 & 942 & $20^{\circ} 15.8^{\prime} \mathrm{N}$ & $59^{\circ} 14.3^{\prime} \mathrm{W}$ & 397.6 \\
\hline 16 & 3 & 1000 & $20^{\circ} 16.4^{\prime} \mathrm{N}$ & $59^{\circ} 11.2^{\prime} W$ & 400.6 \\
\hline 16 & 3 & 1230 & $20^{\circ} 23.0^{\prime} \mathrm{N}$ & $58^{\circ} 45.7^{\prime} \mathrm{W}$ & 425.4 \\
\hline 16 & 3 & 1402 & $20^{\circ} 27.9^{\prime} \mathrm{N}$ & $58^{\circ} 30.4^{\prime} \mathrm{W}$ & 440.5 \\
\hline 16 & 3 & 1506 & $20^{\circ} 31.0^{\prime} \mathrm{N}$ & $58^{\circ} 19.6^{\prime} W$ & 451.1 \\
\hline 16 & 3 & 1548 & $20^{\circ} 33.5^{\prime} \mathrm{N}$ & $58^{\circ} 12.4^{\prime} \mathrm{W}$ & 458.3 \\
\hline 16 & 3 & 1648 & $20^{\circ} 36.9^{\prime} \mathrm{N}$ & $58^{\circ} 2.2^{\prime} \mathrm{W}$ & 468.4 \\
\hline 16 & 3 & 1652 & $20^{\circ} 37.1^{\prime} \mathrm{N}$ & $58^{\circ} 1.5^{\prime} \mathrm{W}$ & 469.1 \\
\hline 16 & 3 & 1916 & $20^{\circ} 42.4^{\prime} \mathrm{N}$ & $57^{\circ} 36.4^{\prime} \mathrm{W}$ & 493.2 \\
\hline 16 & 3 & 1950 & $20^{\circ} 43.7^{\prime} \mathrm{N}$ & $57^{\circ} 30.3^{\prime} \mathrm{W}$ & 499.0 \\
\hline 16 & 3 & 2102 & $20^{\circ} 45.9^{\prime} \mathrm{N}$ & $57^{\circ} 17.4^{\prime} \mathrm{W}$ & 511.3 \\
\hline 16 & 3 & 2212 & $20^{\circ} 47.5^{\prime} \mathrm{N}$ & $57^{\circ} 5.3^{\prime} \mathrm{W}$ & 522.7 \\
\hline 16 & 3 & 2356 & $20^{\circ} 50.4^{\prime} \mathrm{N}$ & $56^{\circ} 47.1^{\prime} \mathrm{W}$ & 540.0 \\
\hline 17 & 3 & 000 & $20^{\circ} 50.5^{\prime} \mathrm{N}$ & $56^{\circ} 46.4^{\prime} \mathrm{W}$ & 540.7 \\
\hline 17 & 3 & 040 & $20^{\circ} 51.9^{\prime} \mathrm{N}$ & $56^{\circ} 39.0^{\prime} \mathrm{W}$ & 547.7 \\
\hline 17 & 3 & 338 & $20^{\circ} 59.0^{\prime} \mathrm{N}$ & $56^{\circ} 6.5^{\prime} \mathrm{W}$ & 578.9 \\
\hline 17 & 3 & 405 & $20^{\circ} 59.7^{\prime} \mathrm{N}$ & $56^{\circ} 1.6^{\prime} \mathrm{W}$ & 583.5 \\
\hline 17 & 3 & 412 & $20^{\circ} 59.8^{\prime} \mathrm{N}$ & $56^{\circ} 0.7^{\prime} \mathrm{W}$ & 584.3 \\
\hline 17 & 3 & 436 & $21^{\circ} 0.4^{\prime} \mathrm{N}$ & $55^{\circ} 56.4^{\prime} \mathrm{W}$ & 588.4 \\
\hline 17 & 3 & 704 & $21^{\circ} 5.7^{\prime} \mathrm{N}$ & $55^{\circ} 30.0^{\prime} \mathrm{W}$ & 613.6 \\
\hline 17 & 3 & 850 & $21^{\circ} 10.0^{\prime} \mathrm{N}$ & $55^{\circ} 11.2^{\prime} \mathrm{W}$ & 631.7 \\
\hline 17 & 3 & 958 & $21^{\circ} 12.8^{\prime} \mathrm{N}$ & $54^{\circ} 59.3^{\prime} \mathrm{W}$ & 643.1 \\
\hline 17 & 3 & 1030 & $21^{\circ} 14.1^{\prime} \mathrm{N}$ & $54^{\circ} 53.6^{\prime} \mathrm{W}$ & 648.6 \\
\hline 17 & 3 & 1146 & $21^{\circ} 16.6^{\prime} \mathrm{N}$ & $54^{\circ} 39.9^{\prime} \mathrm{W}$ & 661.6 \\
\hline 17 & 3 & 1155 & $21^{\circ} 16.8^{\prime} \mathrm{N}$ & $54^{\circ} 38.3^{\prime} \mathrm{W}$ & 663.1 \\
\hline 17 & 3 & 1600 & $21^{\circ} 21.6^{\prime} \mathrm{N}$ & $53^{\circ} 54.2^{\prime} \mathrm{W}$ & 704.4 \\
\hline 17 & 3 & 1854 & $21^{\circ} 26.0^{\prime} \mathrm{N}$ & $53^{\circ} 23.0^{\prime} \mathrm{W}$ & 733.8 \\
\hline 17 & 3 & 2012 & $21^{\circ} 28.6^{\prime} \mathrm{N}$ & $53^{\circ} 9.2^{\prime} \mathrm{W}$ & 746.9 \\
\hline 17 & 3 & 2120 & $21^{\circ} 31.2^{\prime} \mathrm{N}$ & $52^{\circ} 56.6^{\prime} \mathrm{W}$ & 759.0 \\
\hline 17 & 3 & 2306 & $21^{\circ} 34.1^{\prime} \mathrm{N}$ & $52^{\circ} 37.4^{\prime} \mathrm{W}$ & 777.0 \\
\hline 17 & 3 & 2326 & $21^{\circ} 34.6^{\prime} \mathrm{N}$ & $52^{\circ} 33.9^{\prime} \mathrm{W}$ & 780.4 \\
\hline 18 & 3 & 000 & $21^{\circ} 35.7^{\prime} \mathrm{N}$ & $52^{\circ} 27.9^{\prime} \mathrm{W}$ & 786.0 \\
\hline 18 & 3 & 042 & $21^{\circ} 37.1^{\prime} \mathrm{N}$ & $52^{\circ} 20.6^{\prime} \mathrm{W}$ & 793.0 \\
\hline 18 & 3 & 102 & $21^{\circ} 37.6^{\prime} \mathrm{N}$ & $52^{\circ} 17.1^{\prime} W$ & 796.3 \\
\hline 18 & 3 & 110 & $21^{\circ} 37.9^{\prime} \mathrm{N}$ & $52^{\circ} 15.7^{\prime} \mathrm{W}$ & 797.5 \\
\hline 18 & 3 & 230 & $21^{\circ} 41.6^{\prime} \mathrm{N}$ & $52^{\circ} 2.5^{\prime} \mathrm{W}$ & 810.4 \\
\hline 18 & 3 & 250 & $21^{\circ} 42.5^{\prime} \mathrm{N}$ & $51^{\circ} 59.2^{\prime} \mathrm{W}$ & 813.6 \\
\hline 18 & 3 & 616 & $21^{\circ} 53.3^{\prime} \mathrm{N}$ & $51^{\circ} 23.7^{\prime} \mathrm{W}$ & 848.3 \\
\hline 18 & 3 & 800 & $21^{\circ} 58.8^{\prime} \mathrm{N}$ & $51^{\circ} 5.3^{\prime} \mathrm{W}$ & 866.2 \\
\hline 18 & 3 & 828 & $22^{\circ} 0.3^{\prime} \mathrm{N}$ & $51^{\circ} 0.7^{\prime} \mathrm{W}$ & 870.8 \\
\hline 18 & 3 & 910 & $22^{\circ} 2.1^{\prime} \mathrm{N}$ & $50^{\circ} 53.6^{\prime} \mathrm{W}$ & 877.6 \\
\hline 18 & 3 & 1054 & $22^{\circ} 5.0^{\prime} \mathrm{N}$ & $50^{\circ} 36.8^{\prime} \mathrm{W}$ & 893.4 \\
\hline 18 & 8 & 1158 & $22^{\circ} 7.7 ' \mathrm{~N}$ & $50^{\circ} 25.8^{\prime} \mathrm{W}$ & 903.9 \\
\hline 18 & 3 & 1342 & $22^{\circ} 12.0^{\prime} \mathrm{N}$ & $50^{\circ} 7.5^{\prime} \mathrm{W}$ & 921.4 \\
\hline 18 & 3 & 1402 & $22^{\circ} 12.7 ' \mathrm{~N}$ & $50^{\circ} 4.0^{\prime} \mathrm{W}$ & 924.7 \\
\hline 18 & 3 & 1504 & $22^{\circ} 14.2^{\prime} \mathrm{N}$ & $49^{\circ} 53.0^{\prime} \mathrm{W}$ & 935.0 \\
\hline 18 & 3 & 1618 & $22^{\circ} 16.2^{\prime} \mathrm{N}$ & $49^{\circ} 40.2^{\prime} \mathrm{W}$ & 947.1 \\
\hline 18 & 3 & 1652 & $22^{\circ} 17.1^{\prime} \mathrm{N}$ & $49^{\circ} 34.4^{\prime} \mathrm{W}$ & 952.5 \\
\hline 18 & 3 & 2220 & $22^{\circ} 27.1^{\prime} \mathrm{N}$ & $48^{\circ} 39.9^{\prime} \mathrm{W}$ & 1003.9 \\
\hline 18 & 3 & 2245 & $22^{\circ} 27.8^{\prime} \mathrm{N}$ & $48^{\circ} 35.8^{\prime} \mathrm{W}$ & 1007.7 \\
\hline 19 & 3 & 000 & $22^{\circ} 29.4^{\prime} \mathrm{N}$ & $48^{\circ} 23.2^{\prime} \mathrm{W}$ & 1019.5 \\
\hline 19 & 3 & 120 & $22^{\circ} 31.1^{\prime} \mathrm{N}$ & $48^{\circ} 9.8^{\prime} \mathrm{W}$ & 1032.0 \\
\hline 19 & 3 & 250 & $22^{\circ} 32.8^{\prime} \mathrm{N}$ & $47^{\circ} 53.6^{\prime} \mathrm{W}$ & 1047.0 \\
\hline 19 & 3 & 318 & $22^{\circ} 33.3^{\prime} \mathrm{N}$ & $47^{\circ} 48.8^{\prime} \mathrm{W}$ & 1051.5 \\
\hline 19 & 3 & 440 & $22^{\circ} 34.9^{\prime} \mathrm{N}$ & $47^{\circ} 34.8^{\prime} \mathrm{W}$ & 1064.5 \\
\hline 19 & 3 & 1006 & $22^{\circ} 41.9^{\prime} \mathrm{N}$ & $46^{\circ} 38.1^{\prime} \mathrm{W}$ & 1117.3 \\
\hline 19 & 3 & 1220 & $22^{\circ} 44.2^{\prime} \mathrm{N}$ & $46^{\circ} 13.8^{\prime} \mathrm{W}$ & 1139.8 \\
\hline
\end{tabular}


Table 1. (Continued).

\begin{tabular}{ccccccccc}
\hline Date & Month $^{\mathrm{a}}$ & $\begin{array}{c}\text { Time } \\
\text { (GMT) }\end{array}$ & Latitude & Longitude & $\begin{array}{c}\text { Distance } \\
\text { (n. mi.) }\end{array}$ & $\begin{array}{c}\text { Speed } \\
\text { (knots) }\end{array}$ & $\begin{array}{c}\text { Course } \\
\text { (degrees) }\end{array}$ & Comments \\
\hline 19 & 3 & 1230 & $22^{\circ} 443^{\prime} \mathrm{N}$ & $46^{\circ} 12.6^{\prime} \mathrm{W}$ & 1141.0 & 6.3 & 86 & Change speed \\
19 & 3 & 1234 & $22^{\circ} 44.3^{\prime} \mathrm{N}$ & $46^{\circ} 12.1^{\prime} \mathrm{W}$ & 1141.4 & 6.3 & 83 & Satellite navigation \\
19 & 3 & 1252 & $22^{\circ} 44.5^{\prime} \mathrm{N}$ & $46^{\circ} 10.1^{\prime} \mathrm{W}$ & 1143.3 & 6.3 & 81 & Change course \\
19 & 3 & 1320 & $22^{\circ} 450^{\prime} \mathrm{N}$ & $46^{\circ} 6.9^{\prime} \mathrm{W}$ & 1146.3 & 6.2 & 81 & Satellite navigation \\
19 & 3 & 1338 & $22^{\circ} 453^{\prime} \mathrm{N}$ & $46^{\circ} 4.9^{\prime} \mathrm{W}$ & 1148.1 & 6.0 & 80 & Cross over Site 395
\end{tabular}

On station

\begin{tabular}{|c|c|c|c|c|c|c|c|c|}
\hline 31 & 3 & 1857 & $22^{\circ} 40.3^{\prime} \mathrm{N}$ & $46^{\circ} 15.0^{\prime} \mathrm{W}$ & 1158.7 & 6.5 & 74 & Underway \\
\hline 31 & 3 & 1915 & $22^{\circ} 40.9^{\prime} \mathrm{N}$ & $46^{\circ} 13.0^{\prime} \mathrm{W}$ & 1160.6 & 5.7 & 74 & Change speed \\
\hline 31 & 3 & 1920 & $22^{\circ} 41.0^{\prime} \mathrm{N}$ & $46^{\circ} 12.5^{\prime} \mathrm{W}$ & 1161.1 & 5.6 & 74 & Satellite navigation \\
\hline 31 & 3 & 1937 & $22^{\circ} 41.4^{\prime} \mathrm{N}$ & $46^{\circ} 10.9^{\prime} \mathrm{W}$ & 1162.7 & 5.6 & 60 & Change course \\
\hline 31 & 3 & 2028 & $22^{\circ} 43.8^{\prime} \mathrm{N}$ & $46^{\circ} 6.4^{\prime} \mathrm{W}$ & 1167.4 & 5.9 & 53 & Satellite navigation \\
\hline 31 & 3 & 2042 & $22^{\circ} 44.6^{\prime} \mathrm{N}$ & $46^{\circ} 5.2^{\prime} \mathrm{W}$ & 1168.8 & 6.3 & 23 & Change speed \\
\hline 31 & 3 & 2049 & $22^{\circ} 45.3^{\prime} \mathrm{N}$ & $46^{\circ} 4.9^{\prime} \mathrm{W}$ & 1169.5 & 6.1 & 29 & Depart Site 395 \\
\hline 31 & 3 & 2049 & $22^{\circ} 45.3^{\prime} \mathrm{N}$ & $46^{\circ} 4.9^{\prime} \mathrm{W}$ & 1169.5 & 6.1 & 29 & Underway \\
\hline 31 & 3 & 2050 & $22^{\circ} 45.4^{\prime} \mathrm{N}$ & $46^{\circ} 4.8^{\prime} \mathrm{W}$ & 1169.6 & 6.0 & 72 & Change course \\
\hline 31 & 3 & 2216 & $22^{\circ} 48.1^{\prime} \mathrm{N}$ & $45^{\circ} 56.0^{\prime} \mathrm{W}$ & 1178.2 & 6.3 & 72 & Satellite navigation \\
\hline 31 & 3 & 2256 & $22^{\circ} 49.4^{\prime} \mathrm{N}$ & $45^{\circ} 51.7^{\prime} \mathrm{W}$ & 1182.4 & 6.1 & 69 & Satellite navigation \\
\hline 31 & 3 & 2300 & $22^{\circ} 49.5^{\prime} \mathrm{N}$ & $45^{\circ} 51.3^{\prime} \mathrm{W}$ & 1182.8 & 6.6 & 69 & Change speed \\
\hline 1 & 4 & 000 & $22^{\circ} 51.9^{\prime} \mathrm{N}$ & $45^{\circ} 44.6^{\prime} \mathrm{W}$ & 1189.4 & 6.6 & 69 & \\
\hline 1 & 4 & 018 & $22^{\circ} 52.6^{\prime} \mathrm{N}$ & $45^{\circ} 42.6^{\prime} \mathrm{W}$ & 1191.4 & 7.4 & 73 & Satellite navigation \\
\hline 1 & 4 & 040 & $22^{\circ} 53.4^{\prime} \mathrm{N}$ & $45^{\circ} 39.8^{\prime} \mathrm{W}$ & 1194.1 & 7.0 & 72 & Satellite navigation \\
\hline 1 & 4 & 104 & $22^{\circ} 54.3^{\prime} \mathrm{N}$ & $45^{\circ} 36.9^{\prime} \mathrm{W}$ & 1196.9 & 7.0 & 75 & Change course \\
\hline 1 & 4 & 206 & $22^{\circ} 56.2^{\prime} \mathrm{N}$ & $45^{\circ} 29.3^{\prime} \mathrm{W}$ & 1204.2 & 7.3 & 75 & Satellite navigation \\
\hline 1 & 4 & 301 & $22^{\circ} 58.0^{\prime} \mathrm{N}$ & $45^{\circ} 22.3^{\prime} \mathrm{W}$ & 1210.8 & 7.3 & 73 & Change course \\
\hline 1 & 4 & 522 & $23^{\circ} 3.0^{\prime} \mathrm{N}$ & $45^{\circ} 4.6^{\prime} \mathrm{W}$ & 1227.9 & 6.7 & 77 & Satellite navigation \\
\hline 1 & 4 & 539 & $23^{\circ} 3.4^{\prime} \mathrm{N}$ & $45^{\circ} 2.6^{\prime} \mathrm{W}$ & 1229.8 & 7.8 & 76 & Change speed \\
\hline 1 & 4 & 710 & $23^{\circ} 6.2^{\prime} \mathrm{N}$ & $44^{\circ} 50.1^{\prime} \mathrm{W}$ & 1241.6 & 7.5 & 75 & Satellite navigation \\
\hline 1 & 4 & 816 & $23^{\circ} 8.4^{\prime} \mathrm{N}$ & $44^{\circ} 41.4^{\prime} \mathrm{W}$ & 1249.9 & 7.6 & 75 & Satellite navigation \\
\hline 1 & 4 & 1004 & $23^{\circ} 12.0^{\prime} \mathrm{N}$ & $44^{\circ} 27.1^{\prime} \mathrm{W}$ & 1263.5 & 7.6 & 77 & Satellite navigation \\
\hline 1 & 4 & 1140 & $23^{\circ} 14.8^{\prime} \mathrm{N}$ & $44^{\circ} 14.2^{\prime} \mathrm{W}$ & 1275.7 & 7.7 & 74 & Satellite navigation \\
\hline 1 & 4 & 1202 & $23^{\circ} 15.6^{\prime} \mathrm{N}$ & $44^{\circ} 11.2^{\prime} \mathrm{W}$ & 1278.6 & 7.7 & 72 & Change course \\
\hline 1 & 4 & 1326 & $23^{\circ} 18.9^{\prime} \mathrm{N}$ & $44^{\circ} 0.0^{\prime} \mathrm{W}$ & 1289.4 & 7.9 & 72 & Satellite navigation \\
\hline 1 & 4 & 1500 & $23^{\circ} 22.7^{\prime} \mathrm{N}$ & $43^{\circ} 47.2^{\prime} \mathrm{W}$ & 1301.8 & 7.9 & 74 & Change course \\
\hline 1 & 4 & 1520 & $23^{\circ} 23.4^{\prime} \mathrm{N}$ & $43^{\circ} 44.4^{\prime} \mathrm{W}$ & 1304.4 & 7.5 & 73 & Satellite navigation \\
\hline 1 & 4 & 1619 & $23^{\circ} 25.6^{\prime} \mathrm{N}$ & $43^{\circ} 36.7^{\prime} \mathrm{W}$ & 1311.8 & 8.4 & 73 & Change speed \\
\hline 1 & 4 & 1644 & $23^{\circ} 26.6^{\prime} \mathrm{N}$ & $43^{\circ} 33.0^{\prime} \mathrm{W}$ & 1315.3 & 8.2 & 73 & Satellite navigation \\
\hline 1 & 4 & 1830 & $23^{\circ} 30.8^{\prime} \mathrm{N}$ & $43^{\circ} 17.9^{\prime} \mathrm{W}$ & 1329.8 & 8.1 & 72 & Satellite navigation \\
\hline 1 & 4 & 1938 & $23^{\circ} 33.7^{\prime} \mathrm{N}$ & $43^{\circ} 8.4^{\prime} \mathrm{W}$ & 1339.0 & 8.2 & 72 & \\
\hline 1 & 4 & 2124 & $23^{\circ} 38.2^{\prime} \mathrm{N}$ & $42^{\circ} 53.3^{\prime} \mathrm{W}$ & 1353.5 & 8.0 & 76 & Satellite navigation \\
\hline 1 & 4 & 2332 & $23^{\circ} 42.2^{\prime} \mathrm{N}$ & $42^{\circ} 35.2^{\prime} \mathrm{W}$ & 1370.6 & 7.6 & 78 & Satellite navigation \\
\hline 2 & 4 & 000 & $23^{\circ} 43.0^{\prime} \mathrm{N}$ & $42^{\circ} 31.4^{\prime} \mathrm{W}$ & 1374.1 & 7.6 & 78 & \\
\hline 2 & 4 & 116 & $23^{\circ} 45.0^{\prime} \mathrm{N}$ & $42^{\circ} 21.2^{\prime} \mathrm{W}$ & 1383.7 & 8.2 & 75 & Satellite navigation \\
\hline 2 & 4 & 145 & $23^{\circ} 46.0^{\prime} \mathrm{N}$ & $42^{\circ} 17.0^{\prime} \mathrm{W}$ & 1387.7 & 8.2 & 73 & Change course \\
\hline 2 & 4 & 308 & $23^{\circ} 49.4^{\prime} \mathrm{N}$ & $42^{\circ} 5.2^{\prime} \mathrm{W}$ & 1399.0 & 8.1 & 79 & Satellite navigation \\
\hline 2 & 4 & 335 & $23^{\circ} 50.1 \times \mathrm{N}$ & $42^{\circ} 1.3^{\prime} \mathrm{W}$ & 1402.7 & 8.1 & 80 & Change course \\
\hline 2 & 4 & 436 & $23^{\circ} 51.6^{\prime} \mathrm{N}$ & $41^{\circ} 52.4^{\prime} \mathrm{W}$ & 1410.9 & 8.5 & 68 & Satellite navigation \\
\hline 2 & 4 & 620 & $23^{\circ} 57.0^{\prime} \mathrm{N}$ & $41^{\circ} 37.5^{\prime} \mathrm{W}$ & 1425.6 & 8.0 & 75 & Satellite navigation \\
\hline 2 & 4 & 728 & $23^{\circ} 59.4^{\prime} \mathrm{N}$ & $41^{\circ} 27.9^{\prime} \mathrm{W}$ & 1434.7 & 8.4 & 74 & Satellite navigation \\
\hline 2 & 4 & 914 & $24^{\circ} 3.4^{\prime} \mathrm{N}$ & $41^{\circ} 12.2^{\prime} \mathrm{W}$ & 1449.6 & 8.6 & 73 & Satellite navigation \\
\hline 2 & 4 & 1046 & $24^{\circ} 7.2^{\prime} \mathrm{N}$ & $40^{\circ} 58.3^{\prime} \mathrm{W}$ & 1462.8 & 8.2 & 72 & Satellite navigation \\
\hline 2 & 4 & 1232 & $24^{\circ} 11.7^{\prime} \mathrm{N}$ & $40^{\circ} 43.2^{\prime} \mathrm{W}$ & 1477.3 & 8.4 & 72 & Satellite navigation \\
\hline 2 & 4 & 1500 & $24^{\circ} 18.2^{\prime} \mathrm{N}$ & $40^{\circ} 21.6^{\prime} \mathrm{W}$ & 1498.0 & 8.4 & 73 & Change course \\
\hline 2 & 4 & 1740 & $24^{\circ} 24.9^{\prime} \mathrm{N}$ & $39^{\circ} 58.2^{\prime} \mathrm{W}$ & 1520.4 & 8.4 & 69 & Satellite navigation \\
\hline 2 & 4 & 1815 & $24^{\circ} 26.7^{\prime} \mathrm{N}$ & $39^{\circ} 53.2^{\prime} \mathrm{W}$ & 1525.3 & 8.4 & 73 & Change course \\
\hline 2 & 4 & 2028 & $24^{\circ} 32.1^{\prime} \mathrm{N}$ & $39^{\circ} 33.7^{\prime} \mathrm{W}$ & 1543.8 & 8.3 & 75 & Change course \\
\hline 2 & 4 & 2036 & $24^{\circ} 32.4^{\prime} \mathrm{N}$ & $39^{\circ} 32.5^{\prime} \mathrm{W}$ & 1544.9 & 8.7 & 74 & Satellite navigation \\
\hline 2 & 4 & 2057 & $24^{\circ} 33.2^{\prime} \mathrm{N}$ & $39^{\circ} 29.3^{\prime} \mathrm{W}$ & 1548.0 & 8.6 & 76 & Change course \\
\hline 3 & 4 & 000 & $24^{\circ} 39.5^{\prime} \mathrm{N}$ & $39^{\circ} 1.1^{\prime} \mathrm{W}$ & 1574.3 & 8.6 & 76 & \\
\hline 3 & 4 & 014 & $24^{\circ} 40.0^{\prime} \mathrm{N}$ & $38^{\circ} 59.0^{\prime} \mathrm{W}$ & 1576.3 & 8.8 & 85 & Satellite navigation \\
\hline 3 & 4 & 315 & $24^{\circ} 42.3^{\prime} \mathrm{N}$ & $38^{\circ} 29.9^{\prime} \mathrm{W}$ & 1602.9 & 8.8 & 82 & Change course \\
\hline 3 & 4 & 530 & $24^{\circ} 45.0^{\prime} \mathrm{N}$ & $38^{\circ} 8.3^{\prime} \mathrm{W}$ & 1622.7 & 8.1 & 81 & Satellite navigation \\
\hline 3 & 4 & 551 & $24^{\circ} 45.4^{\prime} \mathrm{N}$ & $38^{\circ} 5.2^{\prime} \mathrm{W}$ & 1625.5 & 8.1 & 77 & Change course \\
\hline 3 & 4 & 600 & $24^{\circ} 45.7^{\prime} \mathrm{N}$ & $38^{\circ} 3.9^{\prime} \mathrm{W}$ & 1626.7 & 8.1 & 78 & Change course \\
\hline 3 & 4 & 824 & $24^{\circ} 49.8^{\prime} \mathrm{N}$ & $37^{\circ} 43.0^{\prime} \mathrm{W}$ & 1646.2 & 7.8 & 78 & Satellite navigation \\
\hline 3 & 4 & 845 & $24^{\circ} 50.4^{\prime} \mathrm{N}$ & $37^{\circ} 40.0^{\prime} \mathrm{W}$ & 1648.9 & 7.8 & 75 & Change course \\
\hline 3 & 4 & 1148 & $24^{\circ} 56.5^{\prime} \mathrm{N}$ & $37^{\circ} 14.7^{\prime} \mathrm{W}$ & 1672.7 & 8.4 & 73 & Satellite navigation \\
\hline 3 & 4 & 1600 & $25^{\circ} 7.0^{\prime} \mathrm{N}$ & $36^{\circ} 37.7^{\prime} \mathrm{W}$ & 1707.8 & 8.4 & 77 & Change course \\
\hline 3 & 4 & 1650 & $25^{\circ} 8.6^{\prime} \mathrm{N}$ & $36^{\circ} 30.2^{\prime} \mathrm{W}$ & 1714.8 & 8.0 & 77 & Satellite navigation \\
\hline 3 & 4 & 1838 & $25^{\circ} 11.8^{\prime} \mathrm{N}$ & $36^{\circ} 14.7^{\prime} \mathrm{W}$ & 1729.2 & 7.9 & 77 & Satellite navigation \\
\hline 3 & 4 & 1946 & $25^{\circ} 13.8^{\prime} \mathrm{N}$ & $36^{\circ} 5.0^{\prime} \mathrm{W}$ & 1738.2 & 8.4 & 77 & Satellite navigation \\
\hline 3 & 4 & 2338 & $25^{\circ} 21.2^{\prime} \mathrm{N}$ & $35^{\circ} 30.2^{\prime} \mathrm{W}$ & 1770.5 & 8.1 & 79 & Satellite navigation \\
\hline 4 & 4 & 000 & $25^{\circ} 21.8^{\prime} \mathrm{N}$ & $35^{\circ} 27.0^{\prime} \mathrm{W}$ & 1773.5 & 8.1 & 79 & \\
\hline 4 & 4 & 005 & $25^{\circ} 21.9^{\prime} \mathrm{N}$ & $35^{\circ} 26.2^{\prime} \mathrm{W}$ & 1774.2 & 8.1 & 80 & Change course \\
\hline 4 & 4 & 021 & $25^{\circ} 22.3^{\prime} \mathrm{N}$ & $35^{\circ} 23.9^{\prime} \mathrm{W}$ & 1776.3 & 8.6 & 80 & Change speed \\
\hline 4 & 4 & 442 & $25^{\circ} 28.8^{\prime} \mathrm{N}$ & $34^{\circ} 43.0^{\prime} \mathrm{W}$ & 1813.8 & 8.9 & 80 & Satellite navigation \\
\hline 4 & 4 & 505 & $25^{\circ} 29.4^{\prime} \mathrm{N}$ & $34^{\circ} 39^{\prime} 3^{\prime} \mathrm{W}$ & 1817.2 & 8.9 & 78 & Change course \\
\hline 4 & 4 & 628 & $25^{\circ} 32.0^{\prime} \mathrm{N}$ & $34^{\circ} 26.0^{\prime} \mathrm{W}$ & 1829.5 & 9.0 & 78 & Satellite navigation \\
\hline 4 & 4 & 652 & $25^{\circ} 32.7^{\prime} \mathrm{N}$ & $34^{\circ} 22.1^{\prime} \mathrm{W}$ & 1833.1 & 9.0 & 76 & Change course \\
\hline 4 & 4 & 736 & $25^{\circ} 34.3^{\prime} \mathrm{N}$ & $34^{\circ} 15.0^{\prime} \mathrm{W}$ & 1839.7 & 8.8 & 76 & Satellite navigation \\
\hline 4 & 4 & 924 & $25^{\circ} 38.2^{\prime} \mathrm{N}$ & $33^{\circ} 58.0^{\prime} \mathrm{W}$ & 1855.5 & 8.4 & 76 & Satellite navigation \\
\hline 4 & 4 & 1014 & $25^{\circ} 39.9^{\prime} \mathrm{N}$ & $33^{\circ} 50.5^{\prime} \mathrm{W}$ & 1862.5 & 8.9 & 77 & Satellite navigation \\
\hline 4 & 4 & 1100 & $25^{\circ} 41.5^{\prime} \mathrm{N}$ & $33^{\circ} 43.1^{\prime} \mathrm{W}$ & 1869.3 & 8.1 & 75 & Satellite navigation \\
\hline 4 & 4 & 1200 & $25^{\circ} 43.6^{\prime} \mathrm{N}$ & $33^{\circ} 34.4^{\prime} \mathrm{W}$ & 1877.4 & 8.6 & 76 & Satellite navigation \\
\hline 4 & 4 & 1246 & $25^{\circ} 45.2^{\prime} \mathrm{N}$ & $33^{\circ} 27.3^{\prime} \mathrm{W}$ & 1884.0 & 8.9 & 76 & Satellite navigation \\
\hline 4 & 4 & 1410 & $25^{\circ} 48.2^{\prime} \mathrm{N}$ & $33^{\circ} 13.9^{\prime} \mathrm{W}$ & 1896.5 & 8.9 & 78 & Change course \\
\hline 4 & 4 & 1428 & $25^{\circ} 48.7^{\prime} \mathrm{N}$ & $33^{\circ} 11.0^{\prime} \mathrm{W}$ & 1899.1 & 8.9 & 78 & Satellite navigation \\
\hline 4 & 4 & 1602 & $25^{\circ} 51.5^{\prime} \mathrm{N}$ & $32^{\circ} 55.9^{\prime} \mathrm{W}$ & 1913.0 & 9.1 & 79 & Satellite navigation \\
\hline 4 & 4 & 1627 & $25^{\circ} 52.2^{\prime} \mathrm{N}$ & $32^{\circ} 51.8^{\prime} \mathrm{W}$ & 1916.8 & 9.1 & 80 & Change course \\
\hline 4 & 4 & 1746 & $25^{\circ} 54.3^{\prime} \mathrm{N}$ & $32^{\circ} 38.6^{\prime} \mathrm{W}$ & 1928.8 & 8.8 & 80 & Satellite navigation \\
\hline 4 & 4 & 1856 & $25^{\circ} 56.1^{\prime} \mathrm{N}$ & $32^{\circ} 27.4^{\prime} \mathrm{W}$ & 1939.0 & 8.8 & 80 & Satellite navigation \\
\hline
\end{tabular}


Table 1. (Continued).

\begin{tabular}{|c|c|c|c|c|c|c|c|c|}
\hline Date & Month $^{\mathrm{a}}$ & $\begin{array}{c}\text { Time } \\
\text { (GMT) }\end{array}$ & Latitude & Longitude & $\begin{array}{l}\text { Distance } \\
\text { (n. mi.) }\end{array}$ & $\begin{array}{c}\text { Speed } \\
\text { (knots) }\end{array}$ & $\begin{array}{c}\text { Course } \\
\text { (degrees) }\end{array}$ & Comments \\
\hline 4 & 4 & 2042 & $25^{\circ} 58.7^{\prime} \mathrm{N}$ & $32^{\circ} 10.4^{\prime} \mathrm{W}$ & 1954.6 & 8.8 & 78 & Satellite navigation \\
\hline 4 & 4 & 2200 & $26^{\circ} 1.0^{\prime} \mathrm{N}$ & $31^{\circ} 58.0^{\prime} \mathrm{W}$ & 1965.9 & 8.5 & 79 & Satellite navigation \\
\hline 4 & 4 & 2340 & $26^{\circ} 3.8^{\prime} \mathrm{N}$ & $31^{\circ} 42.5^{\prime} \mathrm{W}$ & 1980.1 & 8.4 & 80 & Satellite navigation \\
\hline 5 & 4 & 000 & $26^{\circ} 4.3^{\prime} \mathrm{N}$ & $31^{\circ} 39.4^{\prime} \mathrm{W}$ & 1982.9 & 8.4 & 80 & \\
\hline 5 & 4 & 036 & $26^{\circ} 5.1^{\prime} \mathrm{N}$ & $31^{\circ} 33.9^{\prime} \mathrm{W}$ & 1988.0 & 8.3 & 79 & Satellite navigation \\
\hline 5 & 4 & 218 & $26^{\circ} 7.7^{\prime} \mathrm{N}$ & $31^{\circ} 18.4^{\prime} \mathrm{W}$ & 2002.1 & 8.8 & 79 & Satellite navigation \\
\hline 5 & 4 & 354 & $26^{\circ} 10.5^{\prime} \mathrm{N}$ & $31^{\circ} 3.0^{\prime} \mathrm{W}$ & 2016.2 & 9.1 & 79 & Satellite navigation \\
\hline 5 & 4 & 538 & $26^{\circ} 13.4^{\prime} \mathrm{N}$ & $30^{\circ} 45.8^{\prime} \mathrm{W}$ & 2031.9 & 9.3 & 80 & Satellite navigation \\
\hline 5 & 4 & 648 & $26^{\circ} 15.3^{\prime} \mathrm{N}$ & $30^{\circ} 33.9^{\prime} \mathrm{W}$ & 2042.8 & 9.3 & 81 & Satellite navigation \\
\hline 5 & 4 & 834 & $26^{\circ} 17.9^{\prime} \mathrm{N}$ & $30^{\circ} 15.8^{\prime} \mathrm{W}$ & 2059.2 & 9.5 & 79 & Satellite navigation \\
\hline 5 & 4 & 908 & $26^{\circ} 18.9^{\prime} \mathrm{N}$ & $30^{\circ} 9.9^{\prime} \mathrm{W}$ & 2064.6 & 9.4 & 79 & Satellite navigation \\
\hline 5 & 4 & 908 & $26^{\circ} 18.9^{\prime} \mathrm{N}$ & $30^{\circ} 9.9^{\prime} \mathrm{W}$ & 2064.6 & 9.4 & 77 & Change course \\
\hline 5 & 4 & 1012 & $26^{\circ} 21.2^{\prime} \mathrm{N}$ & $29^{\circ} 59.0^{\prime} \mathrm{W}$ & 2074.6 & 9.2 & 77 & Satellite navigation \\
\hline 5 & 4 & 1054 & $26^{\circ} 22.7^{\prime} \mathrm{N}$ & $29^{\circ} 52.0^{\prime} \mathrm{W}$ & 2081.1 & 9.5 & 77 & Satellite navigation \\
\hline 5 & 4 & 1150 & $26^{\circ} 24.7^{\prime} \mathrm{N}$ & $29^{\circ} 42.4^{\prime} \mathrm{W}$ & 2089.9 & 9.4 & 77 & Satellite navigation \\
\hline 5 & 4 & 1336 & $26^{\circ} 28.3^{\prime} \mathrm{N}$ & $29^{\circ} 24.3^{\prime} \mathrm{W}$ & 2106.5 & 9.0 & 77 & Satellite navigation \\
\hline 5 & 4 & 1406 & $26^{\circ} 29.3^{\prime} \mathrm{N}$ & $29^{\circ} 19.4^{\prime} \mathrm{W}$ & 2111.0 & 9.0 & 81 & Change course \\
\hline 5 & 4 & 1808 & $26^{\circ} 35.2^{\prime} \mathrm{N}$ & $28^{\circ} 39.2^{\prime} \mathrm{W}$ & 2147.5 & 9.2 & 83 & Satellite navigation \\
\hline 5 & 4 & 1954 & $26^{\circ} 37.3^{\prime} \mathrm{N}$ & $28^{\circ} 21.1^{\prime} \mathrm{W}$ & 2163.8 & 9.4 & 82 & Satellite navigation \\
\hline 5 & 4 & 2204 & $26^{\circ} 40.2^{\prime} \mathrm{N}$ & $27^{\circ} 58.5^{\prime} \mathrm{W}$ & 2184.2 & 9.3 & 81 & Satellite navigation \\
\hline 5 & 4 & 2217 & $26^{\circ} 40.5^{\prime} \mathrm{N}$ & $27^{\circ} 56.3^{\prime} \mathrm{W}$ & 2186.2 & 9.9 & 80 & Change speed \\
\hline 5 & 4 & 2246 & $26^{\circ} 41.3^{\prime} \mathrm{N}$ & $27^{\circ} 51.0^{\prime} \mathrm{W}$ & 2190.9 & 10.3 & 80 & Change speed \\
\hline 5 & 4 & 2330 & $26^{\circ} 42.6^{\prime} \mathrm{N}$ & $27^{\circ} 42.7^{\prime} \mathrm{W}$ & 2198.5 & 10.2 & 79 & Change course \\
\hline 5 & 4 & 2344 & $26^{\circ} 43.0^{\prime} \mathrm{N}$ & $27^{\circ} 40.1^{\prime} \mathrm{W}$ & 2200.9 & 9.9 & 80 & Satellite navigation \\
\hline 6 & 4 & 000 & $26^{\circ} 34.5^{\prime} \mathrm{N}$ & $27^{\circ} 37.2^{\prime} \mathrm{W}$ & 2203.5 & 9.9 & 80 & \\
\hline 6 & 4 & 128 & $26^{\circ} 46.1^{\prime} \mathrm{N}$ & $27^{\circ} 21.2^{\prime} \mathrm{W}$ & 2218.0 & 10.0 & 79 & Satellite navigation \\
\hline 6 & 4 & 348 & $26^{\circ} 50.5^{\prime} \mathrm{N}$ & $26^{\circ} 55.5^{\prime} \mathrm{W}$ & 2241.4 & 10.0 & 81 & Change course \\
\hline 6 & 4 & 600 & $26^{\circ} 53.9^{\prime} \mathrm{N}$ & $26^{\circ} 31.0^{\prime} \mathrm{W}$ & 2263.5 & 9.9 & 83 & Satellite navigation \\
\hline 6 & 4 & 920 & $26^{\circ} 58.2^{\prime} \mathrm{W}$ & $25^{\circ} 54.2^{\prime} \mathrm{W}$ & 2296.6 & 10.0 & 86 & Change course \\
\hline 6 & 4 & 944 & $26^{\circ} 58.5^{\prime} \mathrm{N}$ & $25^{\circ} 49.7^{\prime} \mathrm{W}$ & 2300.6 & 10.5 & 85 & Satellite navigation \\
\hline 6 & 4 & 1130 & $27^{\circ} 0.1^{\prime} \mathrm{N}$ & $26^{\circ} 29.0^{\prime} \mathrm{W}$ & 2319.1 & 10.5 & 83 & Satellite navigation \\
\hline 6 & 4 & 1608 & $27^{\circ} 6.4^{\prime} \mathrm{N}$ & $24^{\circ} 34.6^{\prime} \mathrm{W}$ & 2368.0 & 10.3 & 85 & Satellite navigation \\
\hline 6 & 4 & 1635 & $27^{\circ} 6.8^{\prime} \mathrm{N}$ & $24^{\circ} 29.4^{\prime} \mathrm{W}$ & 2372.6 & 10.4 & 87 & Change course \\
\hline 6 & 4 & 1756 & $27^{\circ} 7.6^{\prime} \mathrm{N}$ & $24^{\circ} 13.7^{\prime} \mathrm{W}$ & 2386.6 & 10.4 & 86 & Satellite navigation \\
\hline 6 & 4 & 1950 & $27^{\circ} 8.9^{\prime} \mathrm{N}$ & $23^{\circ} 51.6^{\prime} \mathrm{W}$ & 2406.3 & 10.4 & 84 & Change course \\
\hline 7 & 4 & 000 & $27^{\circ} 13.2^{\prime} \mathrm{N}$ & $23^{\circ} 3.2^{\prime} \mathrm{W}$ & 2449.5 & 10.4 & 84 & \\
\hline 7 & 4 & 400 & $27^{\circ} 17.3^{\prime} \mathrm{N}$ & $22^{\circ} 16.8^{\prime} \mathrm{W}$ & 2491.0 & 10.1 & 87 & Satellite navigation \\
\hline 7 & 4 & 548 & $27^{\circ} 18.3^{\prime} \mathrm{N}$ & $21^{\circ} 56.4^{\prime} \mathrm{W}$ & 2509.2 & 10.3 & 85 & Satellite navigation \\
\hline 7 & 4 & 610 & $27^{\circ} 18.6^{\prime} \mathrm{N}$ & $21^{\circ} 52.2^{\prime} \mathrm{W}$ & 2513.0 & 10.2 & 83 & Change course \\
\hline 7 & 4 & 836 & $27^{\circ} 21.5^{\prime} \mathrm{N}$ & $21^{\circ} 24.3^{\prime} \mathrm{W}$ & 2537.9 & 10.3 & 82 & Satellite navigation \\
\hline 7 & 4 & 1018 & $27^{\circ} 24.0^{\prime} \mathrm{N}$ & $21^{\circ} 4.7^{\prime} \mathrm{W}$ & 2555.5 & 10.2 & 84 & Satellite navigation \\
\hline 7 & 4 & 1150 & $27^{\circ} 25.6^{\prime} \mathrm{N}$ & $20^{\circ} 47.1^{\prime} \mathrm{W}$ & 2571.2 & 10.2 & 78 & Satellite navigation \\
\hline 7 & 4 & 1306 & $27^{\circ} 28.3^{\prime} \mathrm{N}$ & $20^{\circ} 32.9^{\prime} \mathrm{W}$ & 2584.1 & 10.2 & 83 & Change course \\
\hline 7 & 4 & 1338 & $27^{\circ} 29.0^{\prime} \mathrm{N}$ & $20^{\circ} 26.8^{\prime} \mathrm{W}$ & 2589.5 & 9.9 & 86 & Satellite navigation \\
\hline 7 & 4 & 1455 & $27^{\circ} 29.8^{\prime} \mathrm{N}$ & $20^{\circ} 12.5^{\prime} \mathrm{W}$ & 2602.2 & 9.9 & 88 & Change course \\
\hline 7 & 4 & 1518 & $27^{\circ} 29.9^{\prime} \mathrm{N}$ & $20^{\circ} 8.2^{\prime} \mathrm{W}$ & 2606.1 & 9.9 & 89 & Satellite navigation \\
\hline 7 & 4 & 1547 & $27^{\circ} 30.0^{\prime} \mathrm{N}$ & $20^{\circ} 2.8^{\prime} \mathrm{W}$ & 2610.8 & 9.9 & 90 & Change course \\
\hline 7 & 4 & 1700 & $27^{\circ} 29.9^{\prime} \mathrm{N}$ & $19^{\circ} 49.2^{\prime} \mathrm{W}$ & 2622.9 & 7.9 & 91 & Change speed \\
\hline 7 & 4 & 1704 & $27^{\circ} 29.9^{\prime} \mathrm{N}$ & $19^{\circ} 48.6^{\prime} \mathrm{W}$ & 2623.4 & 8.1 & 89 & Satellite navigation \\
\hline 7 & 4 & 1814 & $27^{\circ} 30.0^{\prime} \mathrm{N}$ & $19^{\circ} 37.9^{\prime} \mathrm{W}$ & 2632.9 & 7.5 & 89 & Satellite navigation \\
\hline 7 & 4 & 1930 & $27^{\circ} 30.1^{\prime} \mathrm{N}$ & $19^{\circ} 27.1^{\prime} \mathrm{W}$ & 2642.5 & 9.5 & 89 & Change speed \\
\hline 7 & 4 & 2002 & $27^{\circ} 30.2^{\prime} \mathrm{N}$ & $19^{\circ} 21.4^{\prime} \mathrm{W}$ & 2647.6 & 9.3 & 89 & Satellite navigation \\
\hline 7 & 4 & 2202 & $27^{\circ} 30.5^{\prime} \mathrm{N}$ & $19^{\circ} 0.4^{\prime} \mathrm{W}$ & 2666.2 & 10.0 & 87 & Satellite navigation \\
\hline 8 & 4 & 000 & $27^{\circ} 31.6^{\prime} \mathrm{N}$ & $18^{\circ} 38.3^{\prime} \mathrm{W}$ & 2685.8 & 10.0 & 87 & \\
\hline 8 & 4 & 130 & $27^{\circ} 32.4^{\prime} \mathrm{N}$ & $18^{\circ} 21.4^{\prime} \mathrm{W}$ & 2700.8 & 10.6 & 85 & Satellite navigation \\
\hline 8 & 4 & 245 & $27^{\circ} 33.6^{\prime} \mathrm{N}$ & $18^{\circ} 6.54^{\prime} \mathrm{W}$ & 2714.1 & 10.6 & 85 & Dead reckoning \\
\hline
\end{tabular}

a of the year 1981 . 

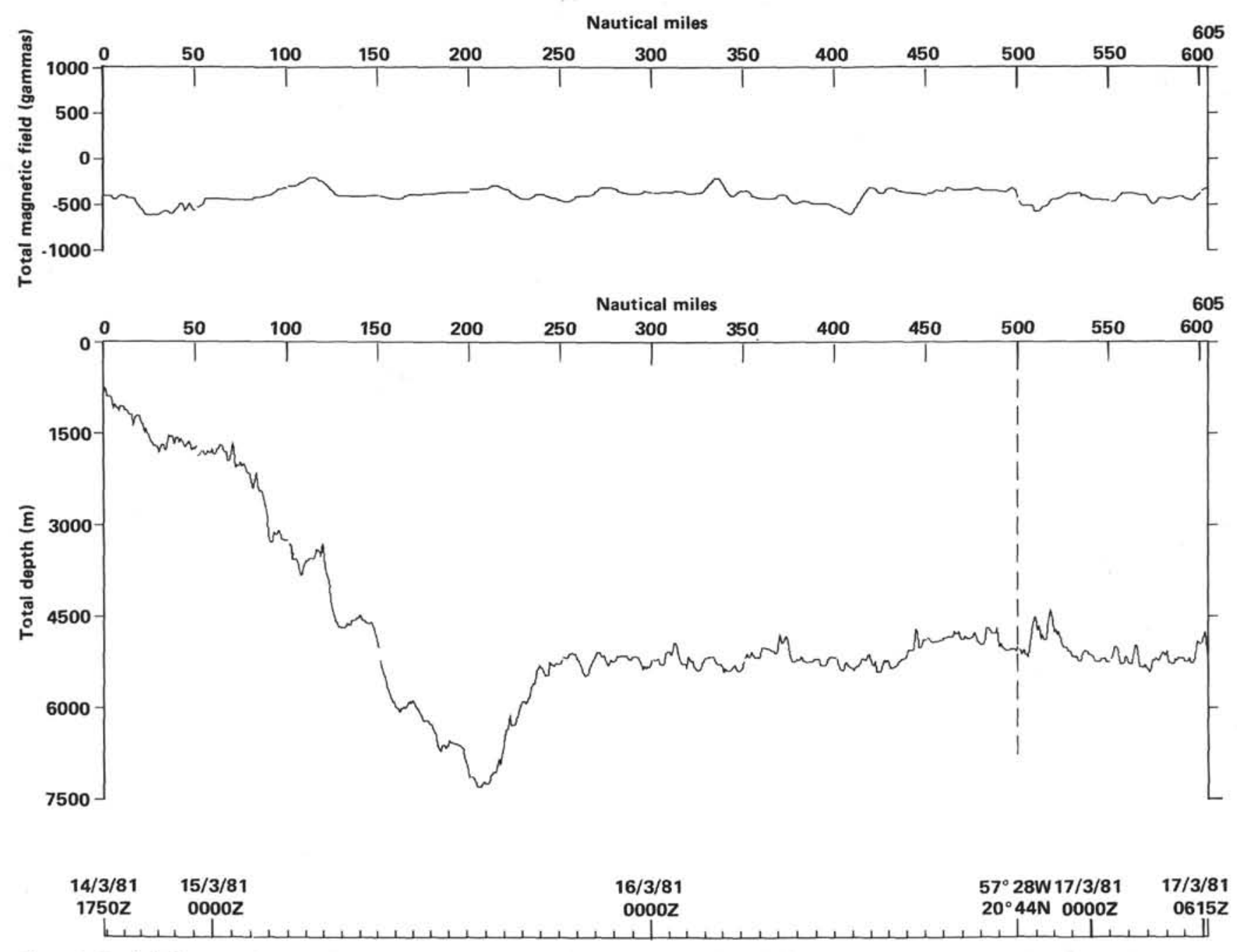

Figure 2. Total-field magnetic anomaly and bathymetry (corrected meters) as a function of distance and steaming time along ship's track shown in Figure 1. 
M. H. SALISBURY, R. D. HYNDMAN, B. LONG
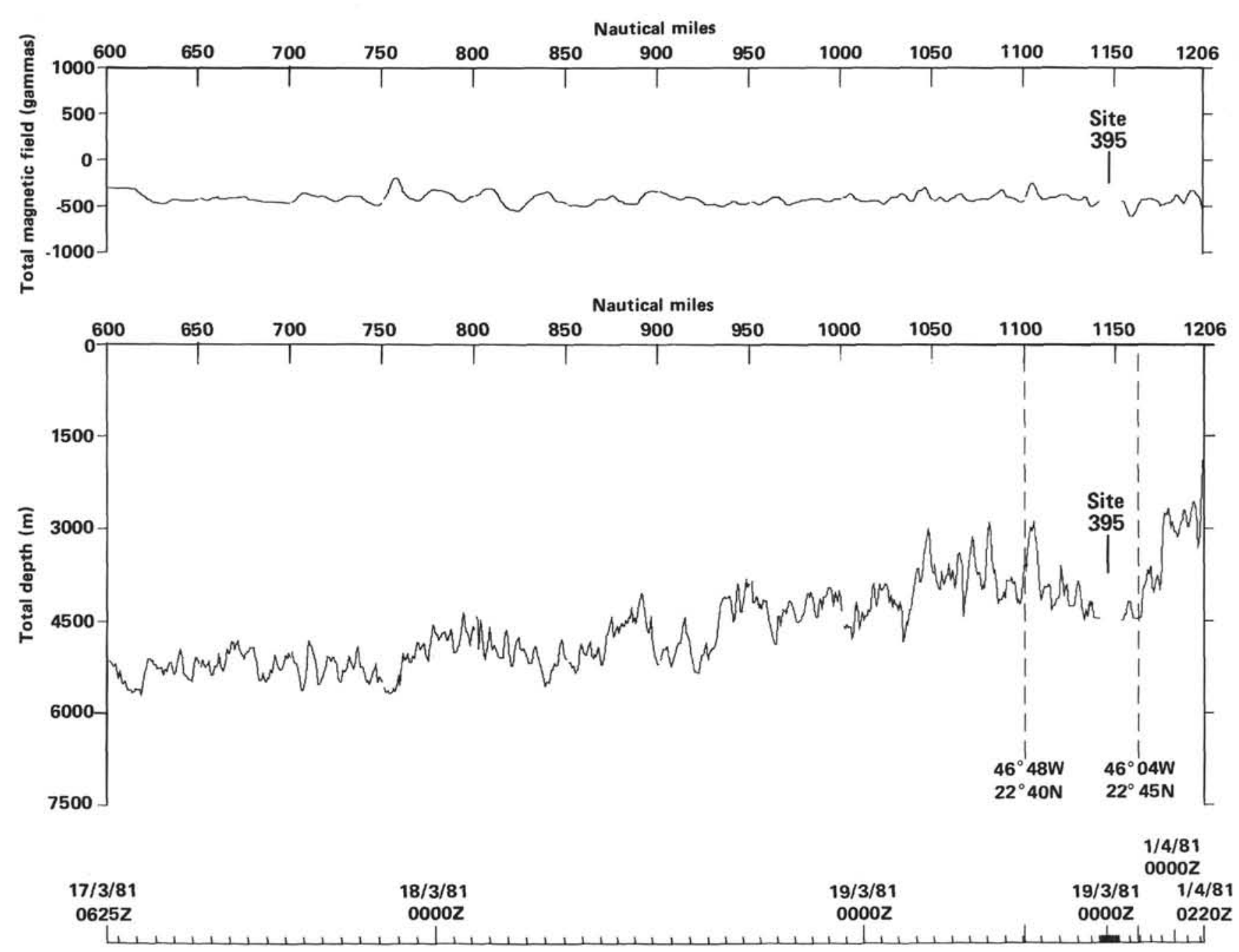

Figure 2. (Continued). 

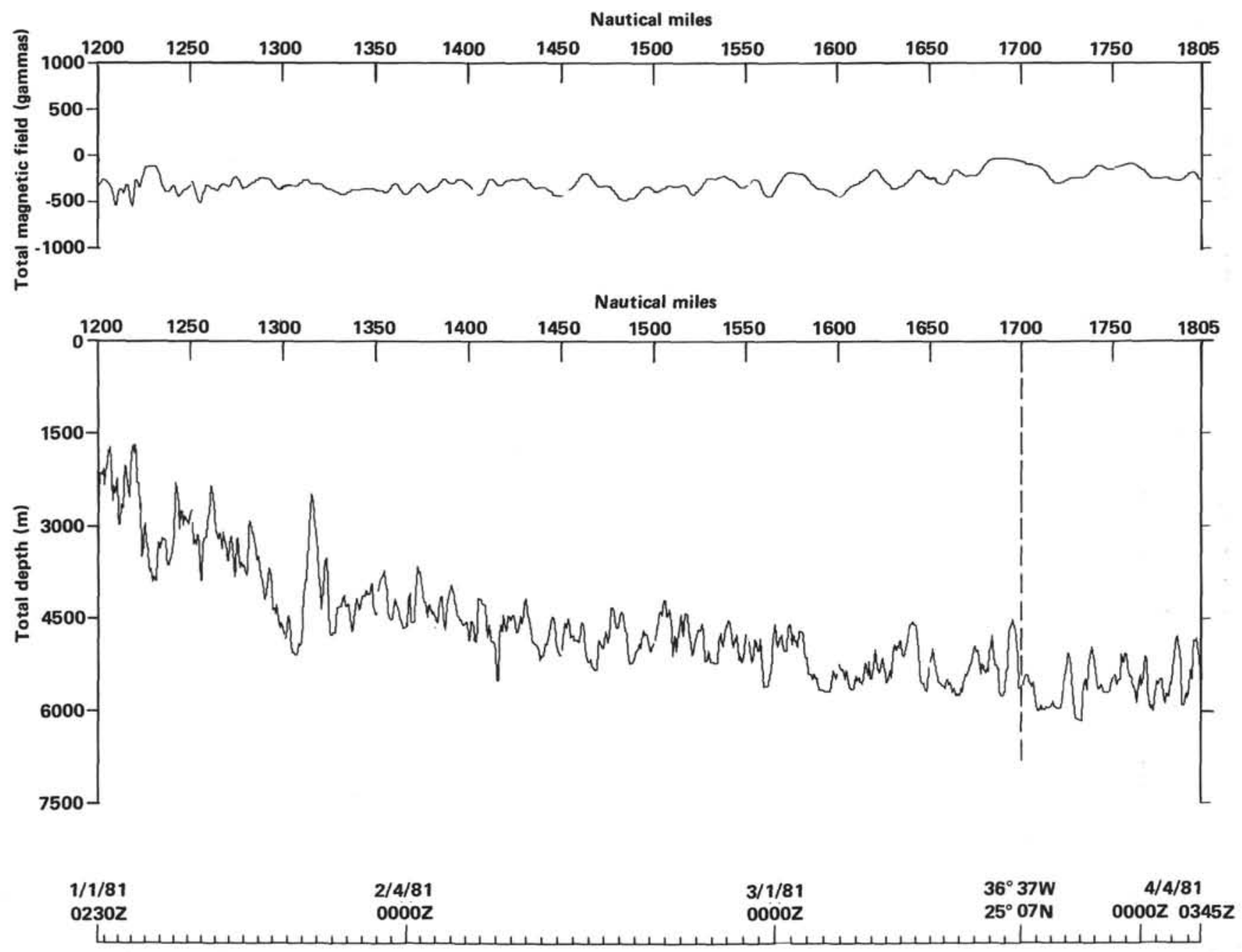

Figure 2. (Continued). 
M. H. SALISBURY, R. D. HYNDMAN, B. LONG
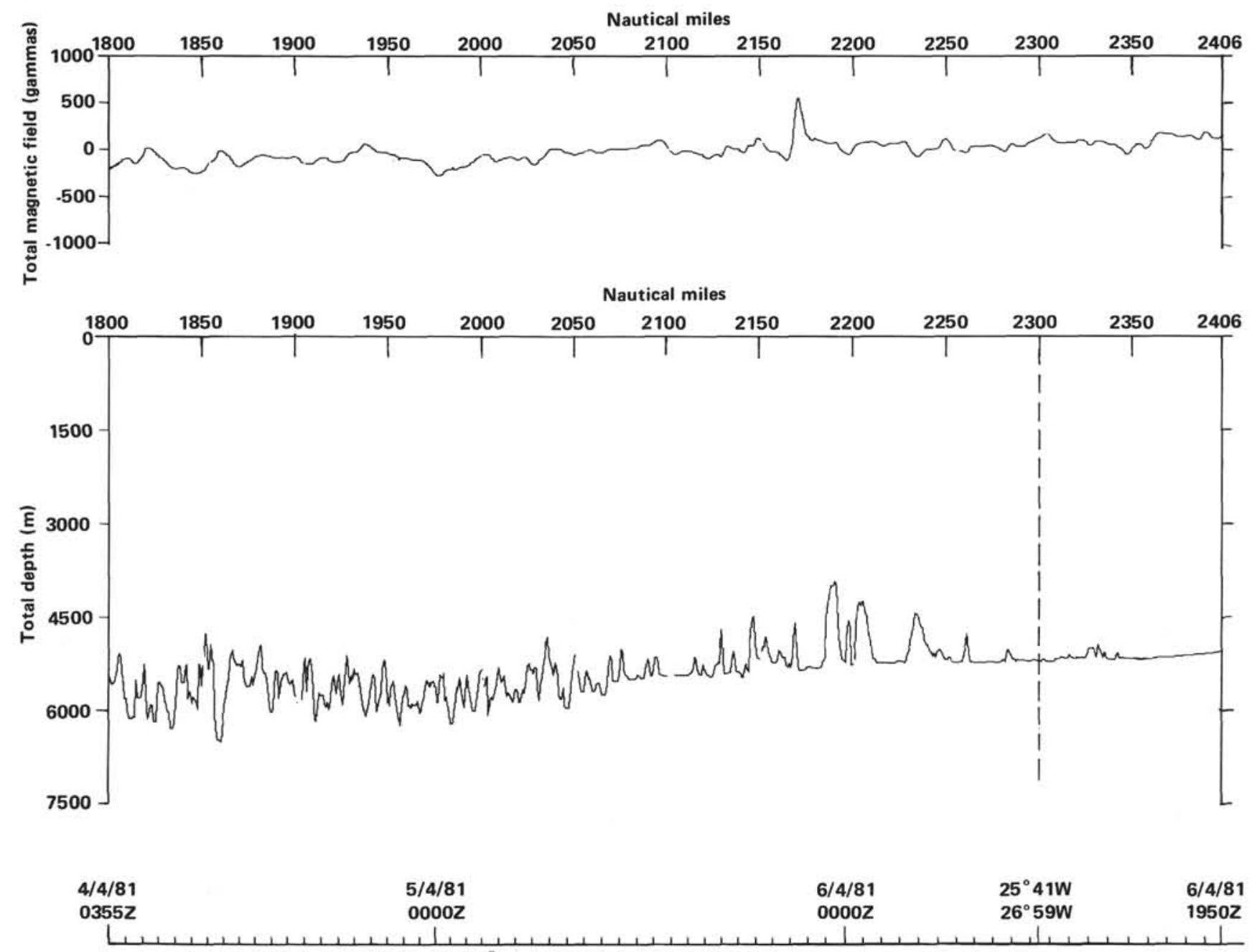

Figure 2. (Continued). 

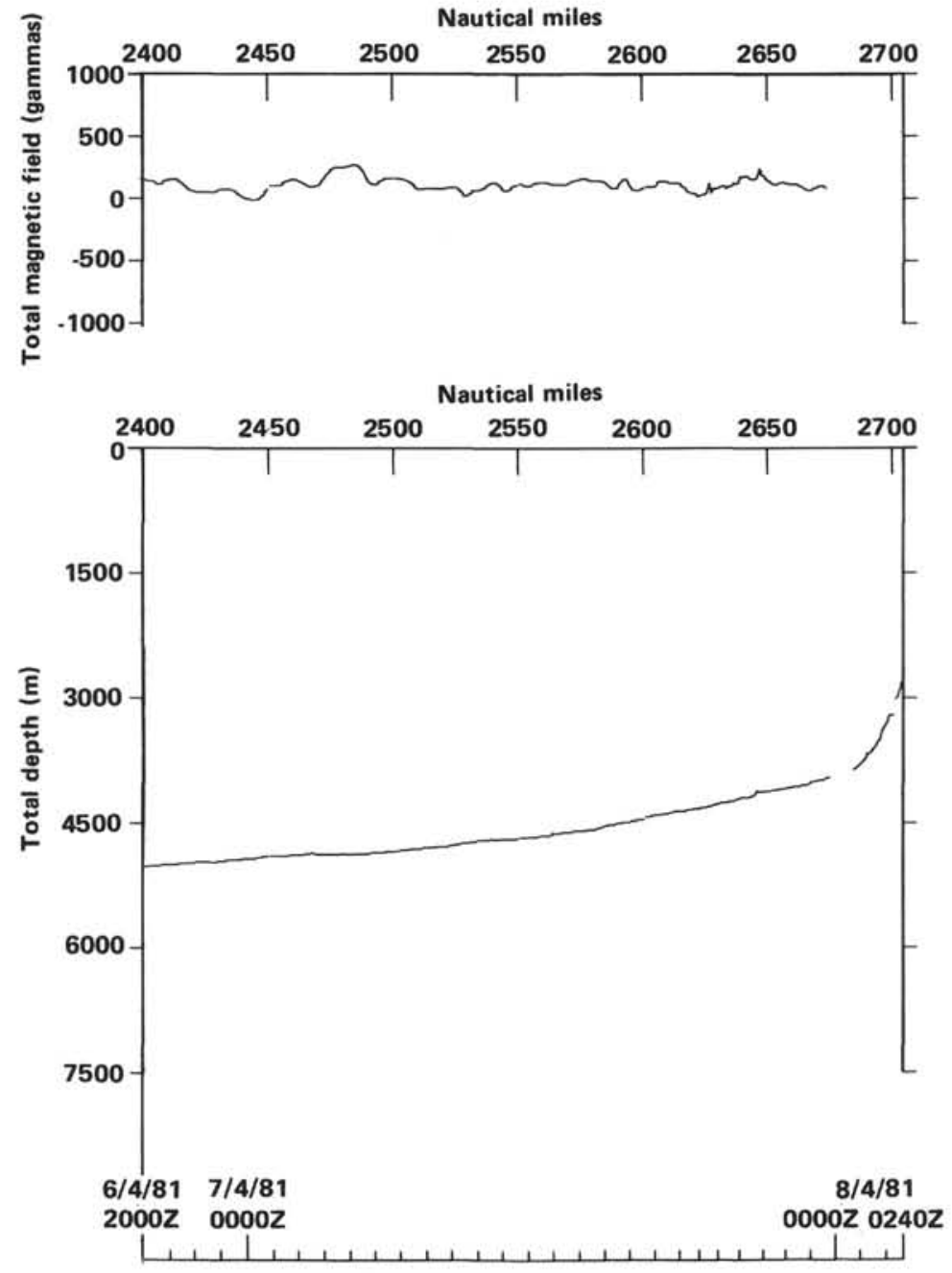

Figure 2. (Continued). 